\title{
VORBEMERKUNG DES HERAUSGEBERS
}

Die Sammlung „Ionenaustauscher in Einzeldarstellungen“ ist durch den Band Nr. 4 der sowjetischen Autoren Laskorin, Smirnowa und Gantman ,Ionenaustauschermembranen und ihre Anwendung" um eine weitere Monografie bereichert worden.

Dank der Übersetzung von G. Diederichs kann dieses wertvolle Buch nunmehr in deutscher Sprache vorgelegt werden.

Historisch gesehen war ohne Zweifel von deutschen Forschern K. F. BovHOEFFER einer der ersten, die - nachdem Kationen- und Anionenaustauscher auf Kunstharzbasis zur Verfügung standen - die besonderen Eigenschaften daraus hergestellter Membranen voraussahen. Mündlich und schriftlich bat er mich wiederholt, ihm doch aus diesem Material hergestellte Membranen für wissenschaftliche Untersuchungen zur Verfügung zu stellen. Leider ließ sich dieser Wunsch nur zögernd befriedigen, da die zunächst allein zur Verfügung stehenden homogenen, gegossenen Membranen mechanisch wenig stabil und kaum versandfähig waren.

Es ist nicht uninteressant, daß BoNHOEFFER und Mitarbeiter bei diesen Studien, die sich an die Arbeiten von Meyer-Sievers, Teorell u. a. anschließen, als Analogon zu einer Anionenaustauschermembran auch ein Dreiphasensystem mit „,oleophilem Elektrolyten“ einsetzten, d. h. einen flüssigen Anionenaustauscher verwandten, was zu einer Erweiterung der Theorie der letztgenannten Forscher führte.

Das Fundament war gelegt; die grundlegenden Unterschiede in den physikalischchemischen Eigenschaften permselektiver und ungeladener Membranen klar erkannt und bestätigt.

Damit eröffneten sich weiterer wissenschaftlicher Forschungsarbeit wie auch der Technik neue interessante Perspektiven und Aufgaben. Ein internationaler Wettlauf setzte ein. Theoretiker wie Praktiker studierten an Ionenaustauschermembranen verschiedenster Provenienz und Herstellung die sich daran abspielenden Vorgänge, zumal die bei der Elektrodialyse eintretenden Reaktionen. Die makromolekulare Chemie empfing neue Impulse. Dem Biologen, Mediziner und Pharmazeuten erschlossen sich tiefere Einblicke auf seinem Fachgebiete. Die Technik bemühte sich, mechanisch stabile und dauerhafte Ionenaustauschermembranen zu schaffen und zu erproben; immer neue praktische bzw. industrielle Anwendungsgebiete wurden erschlossen. 
Das mit großer Sachkenntnis geschaffene Werk der genannten drei Autoren gibt einen ausgezeichneten Überblick über die Entwicklungen auf diesem Sektor bis in die letzten Jahre. Nach kurzer Einführung entwirft Gantman in Kapitel 3 ein knappes Bild unserer heutigen Vorstellungen über Ionenaustauscher; Herstellung und Eigenschaften der verschiedenen Ionenaustauschertypen werden behandelt. Hier hat das letzte Vierteljahrhundert in theoretischer wie in praktischer Hinsicht ohne Zweifel eine gewisse Abrundung gebracht. Dies gilt u. a. auch für die Modellvorstellungen, die man sich von Ionenaustauschern macht.

Sehr ausführlich wird sodann in dem von LASKoRIN und Mitarbeiterin verfaßten Kapitel 4 auf die Herstellung der Ionenaustauschermembranen selbst eingegangen, wobei viele bemerkenswerte, praktische Hinweise gegeben werden.

Das anschließende, wiederum von Gantman geschriebene Kapitel 5 ist den physikalisch-chemischen Eigenschaften der Ionenaustauschermembranen gewidmet und behandelt ausführlich und in kritischer Weise die mannigfachen, in der Literatur empfohlenen Prüfmethoden. Auf die Problematik der Austauschermembranen eingehend, bemüht sich der Verfasser, den komplexen Charakter der an Ionenaustauschermembranen sich abspielenden Vorgänge darzulegen, die nur durch die ,Thermodynamik irreversibler Prozesse" befriedigend wiedergegeben werden können. Auf zahlreiche noch offene Fragestellungen wird hingewiesen, die zum Teil auf die Problematik der Ionenaustauschprozesse neues Licht werfen. Dem Erfinder werden zugleich Anregungen gegeben und aufgezeigt, in welcher Richtung weitere Entwicklungsarbeit sinnvoll anzusetzen ist.

Die anschließenden, wiederum von LAsKorin und SMIRNowa gemeinsam verfaßten drei letzten Kapitel wenden sich sämtlich praktischen Aufgaben zu. Dabei werden zahlreiche, für den Techniker wichtige Winke eingeflochten, die eben nur der geben kann, der selbst wesentlichen Anteil an diesen Entwicklungen genommen hat.

Einem Überblick über die zahlreichen Anwendungsmöglichkeiten von Ionenaustauschermembranen auf technischem und auf wissenschaftlichem Gebiete des Kapitels 6 folgen im 7. Kapitel spezielle Darlegungen, die wesentlich dem Einsatz der Ionenaustauschermembranen im Rahmen der Naßmetallurgie des Urans gewidmet sind. Das letzte Kapitel 8 schließlich behandelt die Kerntechnik einschließlich des wichtigen Gebietes der Beseitigung radioaktiver Abwässer.

In allen diesen Kapiteln geben die Verfasser zunächst einen wertvollen kritischen Uberblick über die vielseitigen Beiträge der internationalen Fachwelt zu dem Gebiete der Ionenaustauschermembranen, um sodann ausführlich auf die eigenen Entwicklungsarbeiten einzugehen.

Ins einzelne gehende Hinweise über zweckmäßge Bauweise und Schaltung elektrodialytischer Apparaturen sowie praktische Erfahrungen über deren Betrieb machen das Ganze zu einem unentbehrlichen Werk für jeden Membran-Praktiker. Aber auch der Leser, der sich über dieses Arbeitsgebiet zu orientieren wünscht, wird den Verfassern außerordentlich dankbar sein für die ungeheure Arbeit, die hier sowohl im Punkte einer Sichtung des Materials wie an praktischer Arbeit geleistet worden ist. 